\title{
Endothelial-to-mesenchymal transition in anticancer therapy and normal tissue damage
}

\author{
Kyu Jin Choi ${ }^{1}$, Jae-Kyung Nam', Ji-Hee Kim', Seo-Hyun Choi ${ }^{2}$ and Yoon-Jin Lee
}

\begin{abstract}
Endothelial-to-mesenchymal transition (EndMT) involves the phenotypic conversion of endothelial-to-mesenchymal cells, and was first discovered in association with embryonic heart development. EndMT can regulate various processes, such as tissue fibrosis and cancer. Recent findings have shown that EndMT is related to resistance to cancer therapy, such as chemotherapy, antiangiogenic therapy, and radiation therapy. Based on the known effects of EndMT on the cardiac toxicity of anticancer therapy and tissue damage of radiation therapy, we propose that EndMT can be targeted as a strategy for overcoming tumor resistance while reducing complications, such as tissue damage. In this review, we discuss EndMT and its roles in damaging cardiac and lung tissues, as well as EndMT-related effects on tumor vasculature and resistance in anticancer therapy. Modulating EndMT in radioresistant tumors and radiationinduced tissue fibrosis can especially increase the efficacy of radiation therapy. In addition, we review the role of hypoxia and reactive oxygen species as the main stimulating factors of tissue damage due to vascular damage and EndMT. We consider drugs that may be clinically useful for regulating EndMT in various diseases. Finally, we argue the importance of EndMT as a therapeutic target in anticancer therapy for reducing tissue damage.
\end{abstract}

\section{Introduction}

Endothelial-to-mesenchymal transition (EndMT) was initially observed by electron microscopy in 1975 during a detailed analysis of endocardium differentiation during the formation of heart valves in vertebrate embryogenesis ${ }^{1}$. This process has also been observed in pathological contexts, such as vascular calcification ${ }^{2}$, atherosclerosis ${ }^{3}$, and cardiac and pulmonary fibrosis ${ }^{4}$. Endothelial cells (ECs) display high plasticity, particularly under pathological conditions and in EndMT. During EndMT, endothelial cells lose their characteristic markers, such as CD31, VE cadherin, Tie2, and vWF, while gaining increased expression of mesenchymal markers, such as fibroblast-specific protein-1 (FSP1), alpha 2 smooth muscle actin ( $\alpha$-SMA), and type I/III collagen.

Correspondence: Yoon-Jin Lee (yjlee8@kirams.re.kr)

${ }^{1}$ Division of Radiation Biomedical Research, Korea Institute of Radiological \& Medical Sciences, Seoul 139-706, Korea

${ }^{2}$ Department of Surgery, Memorial Sloan Kettering Cancer Center, New York, NY 10065, USA

These authors contributed equally: Kyu Jin Choi, Jae-Kyung Nam
Transforming growth factor (TGF)- $\beta 1$ may induce a phenotypic transformation of proliferating endothelial cells into fibroblast-like cells. TGF- $\beta 1$, TGF- $\beta 2$, and bone morphogenetic proteins are also well-known to cause EndMT $^{5,6}$. Several groups have reported on signaling systems and molecules that induce EndMT, including Wnt $/ \beta$-catenin ${ }^{7}$ and Notch signaling ${ }^{8}$, as well as hypoxia ${ }^{9}$ and oxidative stress ${ }^{10}$. These signaling pathways are related to transcription factors involved in mesenchymal transition, such as Snail, Slug, ZEB1, and ZEB2 ${ }^{11,12}$. EndMT exhibits phenotypic changes similar to the epithelial-mesenchymal transition (EMT), which is considered the main process driving phenotypic changes of cells into mesenchymal cells. TGF- $\beta$ also plays important roles in the EMT process in the tumor microenvironment during tumorigenesis and metastases, and in fibroblastic changes of epithelial cells that occur during the development of fibrotic diseases. EndMT has been further studied in atherosclerosis and tumor angiogenesis ${ }^{13,14}$. Recently, increasing evidence has suggested that EndMT-related processes are important contributors to the 
microenvironmental plasticity of cancerous tumors ${ }^{4}$. EndMT leads to the production of cancer-associated fibroblasts $(\mathrm{CAFs})^{15}$, which affect tumor growth and metastases. ECs can differentiate via EndMT into adipocytes and mural cells, such as pericytes and smooth muscle cells ${ }^{16}$. It has also been reported that endothelial progenitor cells differentiate into smooth muscle-like progenitor cells via TGF- $\beta 1$-driven EndMT ${ }^{17}$. Moreover, tumor EndMT induces the formation of both a CAFassociated tumor microenvironment and aberrant tumor vessels $^{18,19}$. Here, we review the role of EndMT in tissue fibrosis and cancer. We focus on EndMT in lung and cardiac tissue, which are often injured during anticancer therapy. In addition, we discuss the therapeutic advantages of targeting EndMT in anticancer therapy.

\section{EndMT effects on tissue damage EndMT in cardiac tissue damage}

Cardiac fibrosis is a common feature in patients with advanced heart failure. Anti-fibrotic therapy may be useful for improving the function of a diseased heart, but the development of therapies has been limited by the incomplete understanding of the origin of fibroblasts in the heart. Cardiac fibrosis occurs because of excessive deposition of the extracellular matrix, which is mediated by the reduction of microcirculation and destruction of the normal myocardial structure. Cardiac fibrosis is associated with the appearance of EndMT ${ }^{20}$. TGF- $\beta 1$ caused EndMT in Tie1Cre;R26RstoplacZ mice (in which the endothelial origin is marked by lacZ expression) and FSP1-GFP mice (expressing green flourescent protein (GFP) under the promoter activity of FSP1), whereas bone morphogenetic protein-7 preserved the endothelial phenotype and inhibited cardiac fibrosis in mice with pressure overload and chronic allograft rejection (Table 1$)^{20}$. EndMT also occurs during the neohypertensive reaction following grafting of veins into the arterial circulation in both mice and humans. An intervening vein graft was evaluated in different mouse models, including a constitutive Endotrack ${ }^{\text {YFP }}$ model and an Endotrack ${ }^{\text {LacZ }}$ and tamoxifen-induced Endotrack ${ }^{\mathrm{YFP}}$ transgenic mouse model. Staining for endothelial-specific markers revealed that in neointimal (but not luminal) $\mathrm{YFP}^{+}$cells, the expression of endothelial-specific markers was lost after grafting. In addition, the expression of the vascular smooth muscle cell markers, SMA and smooth muscle protein 22-alpha (SM22a), progressively increased in $\mathrm{YFP}^{+}$cells (Table 1). Previous data revealed TGF$\beta-$ Smad2/3-Slug signaling as a principal pathway regulating EndMT during vein graft remodeling ${ }^{21}$. A study using ET- ${ }^{\mathrm{f} / \mathrm{f}} ; \mathrm{Tie} 2-\mathrm{Cre}$ transgenic mice with specific ET-1 inhibition showed that diabetes mellitus-induced cardiac fibrosis was associated with the emergence of fibroblasts, and that ET-1 further promoted cardiac fibrosis and heart failure through the accumulation of fibroblasts via EndMT (Table 1) ${ }^{22}$. It has been reported that hypoxic conditions can induce EndMT in cardiac damage. Hypoxia induced EndMT in human coronary endothelial cells, an effect mediated by hypoxia-inducible factor (HIF)- $1 \alpha$-induced activation of SNAIL, an EndMT master-regulatory transcriptional factor ${ }^{23}$.

\section{EndMT in lung injury}

Recently, EndMT was reported to play a role in pulmonary fibrosis. Chronic hypoxia is an important contributing factor to pulmonary hypertension. EndMT was observed in the pulmonary arteries of rat models of pulmonary hypertension. In pulmonary microvascular ECs, hypoxia-induced HIF- $1 \alpha$ activity modulates the transdifferentiation of ECs via Twist ${ }^{24}$. It has been reported that vildagliptin (a dipeptidyl peptidase 4 [DPP-4] inhibitor that serves as an antidiabetic drug) improves vascular dysfunction. In a model of lipopolysaccharide-induced septic lung injury, vildagliptin reduced pulmonary fibrosis by attenuating EndMT (Table 1$)^{25}$. Recent data demonstrated that the mesenchymal transition of pulmonary capillary ECs served as the origin of fibroblasts found during bleomycin-induced pulmonary fibrosis using the Tie2-Cre/CAG-CAT-LacZ double-transgenic mouse system, which enabled tracing of LacZ expression in Tie2positive $\mathrm{ECs}^{26}$. Cotreatment with TGF- $\beta$ and activated Ras caused a persistent EndMT phenotype, inducing

Table 1 The effects of miRNAs on EndMT.

\begin{tabular}{llll}
\hline miRNA & Target & Function & Research model \\
\hline miR-200b & TGF- $\beta$-dependent Smad2/Snail1 & Inhibit EndMT & Retina in diabetic animals \\
miR-18a-5p & Notch2 & Attenuate EndMT & Diabetic cardiomyopathy \\
miR-126a-5p & TGF- $\beta$ signaling & Increase EndMT & Neonatal pulmonary hypertension \\
miR-302c & Metadherin & Inhibit EndMT & Hepatocellular carcinoma \\
miR-199a-5p & Snail/miR-199a-5p axis & Promote EndMT via the & Irradiated human umbilical vein \\
& & Snail/miR-199a-5 $p$ axis & endothelial cells \\
\hline
\end{tabular}


$\alpha$-SMA expression, which was not induced by TGF- $\beta$ or activated Ras alone. The data showed that, via TGF- $\beta$ signaling, Ras/MAPK regulated the completion of EndMT in bleomycin-induced pulmonary fibrosis ${ }^{27}$. Nintedanib is a tyrosine kinase inhibitor that targets platelet-derived growth factor (PDGF) and the fibroblast growth factor receptor, and reduces vascular remodeling-related neointimal lesions and the medial wall thickness of pulmonary arteries. Moreover, Twist expression and related EndMT were reduced by treatment with nintedanib ${ }^{28}$.

\section{Role of oxidative stress and hypoxia in EndMT}

Reactive oxygen species (ROS) have been reported to induce EndMT during oxidative stress ${ }^{10,29}$. Oxidative stress promotes TGF- $\beta 1$ secretion, a key inducer of EndMT ${ }^{30}$. Oxidants can directly activate latent TGF- $\beta^{31}$. In addition, many oxidants can activate the nuclear factorkappa B (NF-kB) pathway ${ }^{32}$, and subsequently induce the production of inflammatory cytokines, which directly stimulate EndMT ${ }^{33,34}$.

Recent reports have shown that EndMT contributes to fibroblast accumulation during heart and kidney fibrosis and cancer ${ }^{6,13,35}$. Although ROS, TGF- $\beta$, inflammatory cytokines, and hypoxia are known to be involved in the development of chronic fibrosis in the lungs, the precise mechanism underlying the progression of initial radiation injuries to chronic fibrosis remains unclear ${ }^{36,37}$. It has also been demonstrated that radiation-induced hypoxia can trigger EndMT through HIF-1 $\alpha$-mediated activation of TGF- $\beta 1 /$ Smad signaling in human pulmonary artery ECs. Moreover, regulating EndMT has been suggested as an effective strategy for stopping radiation-induced pulmonary fibrosis at an early stage ${ }^{38}$. Cells derived from EndMT were previously found during atherosclerotic development in intimal plaques, and this process was guided by TGF- $\beta$ signals, oxidative stress, and hypoxia in vitro and in vivo in mice ${ }^{39}$.

Salvianolic acid A (which has been widely used for the clinical therapy of various diseases involving vascular disturbance) upregulates CD31 and downregulates $\alpha$ SMA, and can attenuate EndMT, suppresses oxidative stress, and alleviates pulmonary vascular remodeling. The $\mathrm{Nrf2/HO}-1$ signaling pathway may be involved in these processes $^{40}$.

The inhibition of ROS production in ECs reduces oxidative stress-induced EndMT. Brain and muscle ARNTlike protein-1 (BMAL1), an essential clock transcription activator, can prevent the accumulation of intracellular ROS caused by lipoproteins, and subsequent EndMT in human endothelial aortic cells ${ }^{41-43}$ in vitro. In addition, BMAL1 deficiency aggravated intracellular ROS accumulation and EndMT progression via bone morphogenetic protein-mediated signaling. These results provide a mechanism to explain the central role of BMAL1 in atherosclerosis progression, and the phenotype switch of plaque cells ${ }^{43}$. Low-intensity pulsed ultrasound protects endothelial human aortic cells from oxidative stressinduced EndMT, which has been associated with PI3K/ AKT signaling ${ }^{44}$. As previously reported, EndMT occurs when oxidative stress and NF- $\mathrm{kB}$ activity are increased in ECs by TGF- $\beta 1$ and TGF- $\beta 2^{45,46}$. Taken together, these findings suggest that inflammation, hypoxia, and endothelial oxidative stress exacerbate EndMT by inducing canonical TGF- $\beta$ signaling ${ }^{30}$. NADPH oxidases and ROS play key roles in mediating fibrotic responses induced by TGF- $\beta$ via Smad2/Smad3 activation ${ }^{30}$. Oxidative stress can induce EndMT conversion via TGF- $\beta 1$ - and TGF- $\beta 2$ dependent pathways and the intracellular ALK5/Smad3/ NF-kB pathway ${ }^{30,47}$.

Recent findings have also suggested that EndMT can be regulated using nanoparticles or natural compounds. Polyglucose sorbitol carboxymethyether-modified $\mathrm{Fe}_{2} \mathrm{O}_{3}$ (PSC- $\mathrm{Fe}_{2} \mathrm{O}_{3}$ ) iron oxide nanoparticles decreased the expression of the endothelial markers CD31 and VE cadherin at an acutely noncytotoxic concentration, and increased the expression of the mesenchymal marker $\alpha$-SMA. FSP, via its peroxidase-like activity, enhanced EC migration and inhibited angiogenic function, which clearly indicated the occurrence of EndMT in $\mathrm{ECs}^{30}$. EndMT leads to vascular damage during angiotensin-IItreated chronic inflammation, which can be prevented by treatment with schisandrin B. Schisandrin B is a natural product derived from Schisandra chinensis that can inhibit the activation of NF-kB. Schisandrin B was also found to suppress inflammation/ROS-mediated EndMT by inhibiting NF- $\mathrm{kB}^{48}$. Hypoxia is the main factor promoting the occurrence of EndMT. The relationship between hypoxia and TGF- $\beta$ signaling is regulated by the expression of microRNAs (miRNAs). miR-126a-5p, which inhibits TGF- $\beta$ signaling, was upregulated in hypoxiainduced persistent pulmonary hypertension of newborns as a cardiac syndrome (Table 2$)^{49}$. Chronic hypoxia increased oxygen consumption and activated fibroblasts in cardiac fibrosis, resulting in aberrant ventricular remodeling ${ }^{50}$. Under hypoxic conditions, the EndMT of human cardiac microvascular ECs promoted tube formation. Autophagy provides protective effects against the EndMT of human cardiac microvascular ECs by degrading Snail under hypoxic conditions ${ }^{51}$. In addition, it has been suggested that hypoxia induces EndMT in human coronary ECs via Hif1a-activated Snail, indicating that endocardium-derived ECs undergo EndMT ${ }^{23}$.

\section{Effects of EndMT on cancer}

EndMT is a specific mechanism resulting in CAF accumulation; antiangiogenic tumor therapy may directly decrease the abundance of activated fibroblasts that are likely to promote cancer progression ${ }^{52}$. 


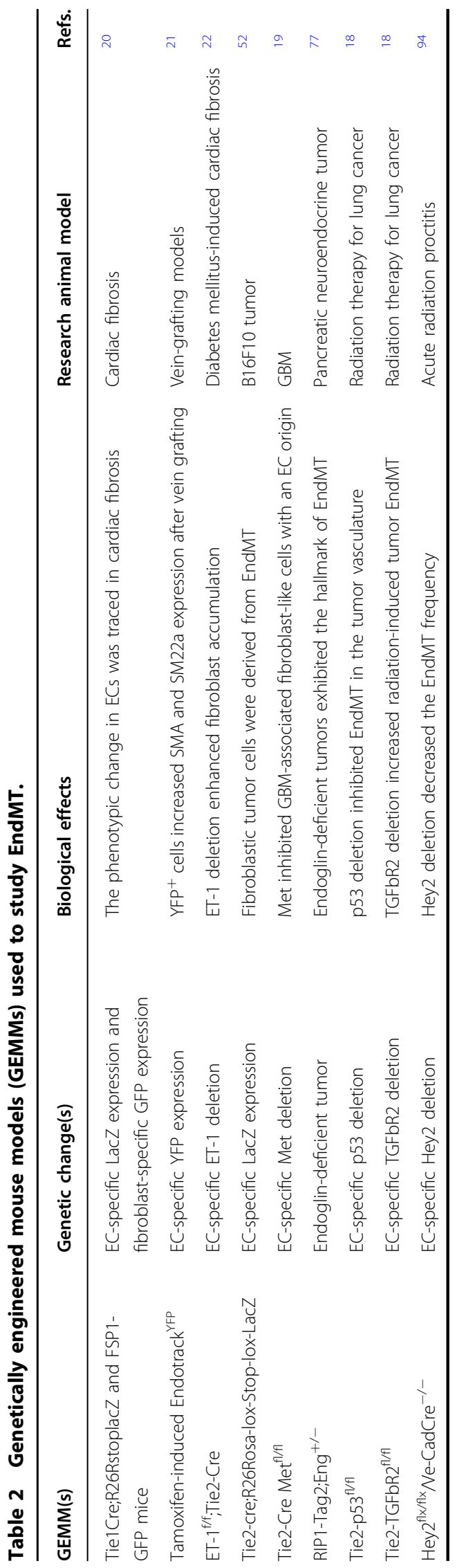

\section{Tumor EndMT and CAFs}

ECs contribute to the abundance of CAFs through EndMT in the tumor microenvironment ${ }^{52}$. Using Tie2cre;R26Rosa-lox-Stop-lox-LacZ transgenic mice, it was discovered that approximately $30 \%$ of fibroblastic cells $\left(\mathrm{FSP}^{+}\right.$cells) and $12 \%$ of $\alpha-\mathrm{SMA}^{+}$cells in the B16F10 tumor stroma were derived from EndMT (Table 1$)^{52}$. Cancer cells can induce EndMT via TGF- $\beta$ through several mechanisms. In hepatocellular carcinoma, miR302c inhibits tumor growth through metadherin, a factor that contributes to cell motility (Table 2$)^{4,53}$. The levels of miR-302c expressed by ECs isolated from tumor tissues were significantly lower than the corresponding levels in normal liver tissues ${ }^{53}$. The levels of miR-302c in ECs correlated negatively with the proliferation rate of the hepatocellular carcinoma cell line $\mathrm{HCCLM}^{53}$.

Tumor-induced EndMT is mediated by factors secreted from tumor cells, such as TGF- $\beta 2$ and interleukin (IL)- $1 \beta$. Tumor-driven EndMT is accompanied by the activation of proinflammatory pathways in $\mathrm{ECs}^{54}$. The expression of cyclooxygenase-2, intercellular adhesion molecule-1, and vascular cell adhesion molecule- 1 is increased, and NF- $\mathrm{kB}$ is activated in EndMT-transformed $\mathrm{ECs}^{3}$. ECs showed phenotypic changes consistent with EndMT when cocultured with OE33 esophageal adenocarcinoma cells expressing high levels of IL- $1 \beta$ and TGF- $\beta 2$. CAFs, which were likely a result of EndMT, were found at the invasive front of esophageal adenocarcinoma, indicating the significance of EndMT in tumor progression ${ }^{54}$. Notably, a remarkably large number of these EndMT-derived CAFs were located close to the invasive tumor front ${ }^{3}$. ECs undergoing tumor-induced EndMT express higher levels of the vascular endothelial growth factor (VEGF) gene, whereas VEGF receptor 2 (VEGFR2) was downregulated in $\mathrm{ECs}^{3}$. EndMT-transformed esophageal ECs may be an important source of VEGF in the tumor microenvironment, and function more in a paracrine than in an autocrine manner ${ }^{54}$.

Loss of Tie-1, an EC-specific receptor essential for the vascular system, induces EndMT in human ECs and pancreatic tumors. Downregulation of Tie-1 triggers EndMT by activating the Slug promoter ${ }^{55}$. EndMT plays an important role in cancer progression and metastasis. ECs that undergo EndMT are more invasive, as they lose expression of their endothelial markers (CD31, von Willebrand factor VIII, and VE cadherin) and acquire a mesenchymal phenotype and an increased migration ability. The tumor promotes a mesenchymal shift in ECs that is regulated by Smad signaling through the synergistic stimulation of TGF- $\beta$ and Notch pathways in breast cancer cells. Tumor cells increase the mesenchymal phenotypes of ECs, but maintain their endothelial phenotypes. It was shown that tumor-stimulated processes that increase extracellular matrix formation are also 
regulated by activation of the Notch pathway via phosphorylation of TGF- $\beta /$ Smad1/ $/ 5^{56-60}$. HSPB1 has been described as a key regulator of EndMT in lung cancer. Endothelial HSPB1 deficiency in the mesenchymal transition of vascular ECs contributes to lung fibrosis and tumorigenesis ${ }^{61}$.

Osteopontin is a multifunctional phospho-glycoprotein that stimulates angiogenesis in ECs. In colorectal cancer, the presence of osteopontin-integrin $\alpha \mathrm{V} \beta 3$ induces HIF$1 \alpha$ expression via a PI3K/Akt/tuberous sclerosis complex 2-mediated and mTORC1-dependent protein synthesis pathway, which transactivates TCF12 gene expression. These findings indicate that HIF- $1 \alpha$ promotes EndMT by inducing TCF12 ${ }^{62}$. EndMT reversal contributes to the control of chemoresistance, irrespective of the level of soluble TGF- $\beta$ that is present. In a xenograft mouse model of multicellular tumor spheroids containing lung cancer cells and human umbilical vein endothelial cells (HUVECs), GSK-3 $\beta$ inhibition reduced the lung cancer volume by inhibiting EndMT, and had a synergistic anticancer effect on non-small-cell lung cancer cells in combination with gefitinib ${ }^{63}$.

The PLEK2-SHIP2 axis promotes both lung cancer cell migration (via TGF- $\beta /$ PI3K/AKT signaling) and vascular invasion. This suggests that PLEK2 can serve as a valuable prognostic marker and a promising target in non-smallcell lung cancer therapy ${ }^{64}$. Researching functional genes and regulatory mechanisms associated with EndMT is important for revealing the mechanisms driving lung cancer metastasis and improving targeted diagnoses or treatments.

\section{EndMT and the tumor vasculature}

EndMT also plays a major role in the tumor vasculature. ECs undergoing tumor-mediated EndMT show functional alterations, such as greater migratory capacity, higher proliferation rates, and a gain of contractile capacity, whereas the cells lose their angiogenic ability to form capillary-like tubes $^{54}$. In glioblastoma, c-Met-mediated EC plasticity induces mesenchymal transformation to promote EC proliferation and migration, resulting in aberrant vasculature formation and chemoresistance to multiple therapies (Table 1) ${ }^{19}$. EndMT contributes to metastatic extravasation and intravasation. Primary tumor vasculature showing EndMT exhibits the loss of endothelial cell-cell junctions, which causes the transendothelial migration of metastatic cells ${ }^{65}$. For example, Rock was shown to reduce microvascular-endothelial hyperpermeability via the Rho/ROCK/MLC pathway of actin stress-fiber formation ${ }^{66}$. The Rho/RACK pathway rescued tight-junction integrity in brain $\mathrm{ECs}^{67}$. ROCK inhibition prevented tumor endothelial migration ${ }^{68}$. Downregulated ERG and FL1 expression caused EndMT in intratumoral ECs in B16F10 melanoma tumors, indicating the aberrant behavior of ECs in pathological environments ${ }^{69}$.

\section{EndMT in anticancer therapy \\ EndMT and CAF chemoresistance}

After chemotherapy, the activated stromal compartment contributes to the survival of residual cancer cells and tumor resistance ${ }^{70}$. CAFs promote tumor-supportive functions through cytokine and metabolite release. The CD10- and GPR77-positive CAF subset exhibits chemoresistance and poor survival in patients with breast or lung cancer ${ }^{71}$. CAFs may originate from ECs via EndMT, bone marrow-derived cells, adipocytes, and stellate cells ${ }^{72}$. Various reports have shown that CAFs mediate tumor progression and resistance, as well as relapse after chemotherapy $^{14}$.

\section{EndMT and anti-VEGF therapy}

Aberrant vascularization is a characteristic of resistance to chemotherapy. Human glioblastoma multiforme (GBM) tissues show an aberrant vasculature altered by EndMT. In GBM, chemoresistance and cancer progression are related to c-Met-mediated EndMT. EC-specific Met deletion resulted in EndMT, inhibited aberrant vascularization and tumor growth, and prolonged the survival of GBM-bearing mice after temozolomide treatment ${ }^{19}$. In GBM mouse models, PDGF-mediated EndMT decreased VEGFR2 expression via the PDGF/NF- $\mathrm{kB} /$ Snail axis in ECs, and thus induced resistance to anti-VEGF/VEGFR therapy. EC-specific PDGFR-B deletion sensitized tumors to antiVEGF therapy ${ }^{73}$. SMA-positive perivascular cells were evaluated for their resistance to antiangiogenic therapy in vivo in malignant melanoma ${ }^{74}$ and pancreatic neuroendocrine tumor mouse models using RIP1-Tag2 mice $^{75}$. Aberrant $\alpha-\mathrm{SMA}^{+}$perivascular cells were related to increased metastasis in RIP1-Tag2 mice deficient in neural cell adhesion molecule ${ }^{76}$. Endoglin-deficient tumors showed increased $\alpha$-SMA- and NG2-positive cells in the blood vessels, and endoglin-deficient tumor ECs in RIP1-Tag2 mice caused EndMT, which induced Twist expression. Endoglin-deficient tumors exhibited more hepatic metastases than wild-type tumors, but maintained their sensitivity to anti-VEGF therapy, suggesting that the synergistic interaction of endoglin deficiency and VEGF inhibition can be used as a combined therapeutic strategy (Table 1$)^{77}$. EC-specific miR-302c inhibited metadherin expression, reduced EndMT, and suppressed tumor growth in hepatocellular carcinoma. Metadherin and Mir-302c may be useful as antiangiogenic therapies in hepatocellular carcinoma (Table 2) ${ }^{53}$.

\section{Cardiotoxicity of anticancer drugs}

Chemotherapeutic agents, including anthracycline and doxorubicin (Dox), and targeted therapies in anticancer 


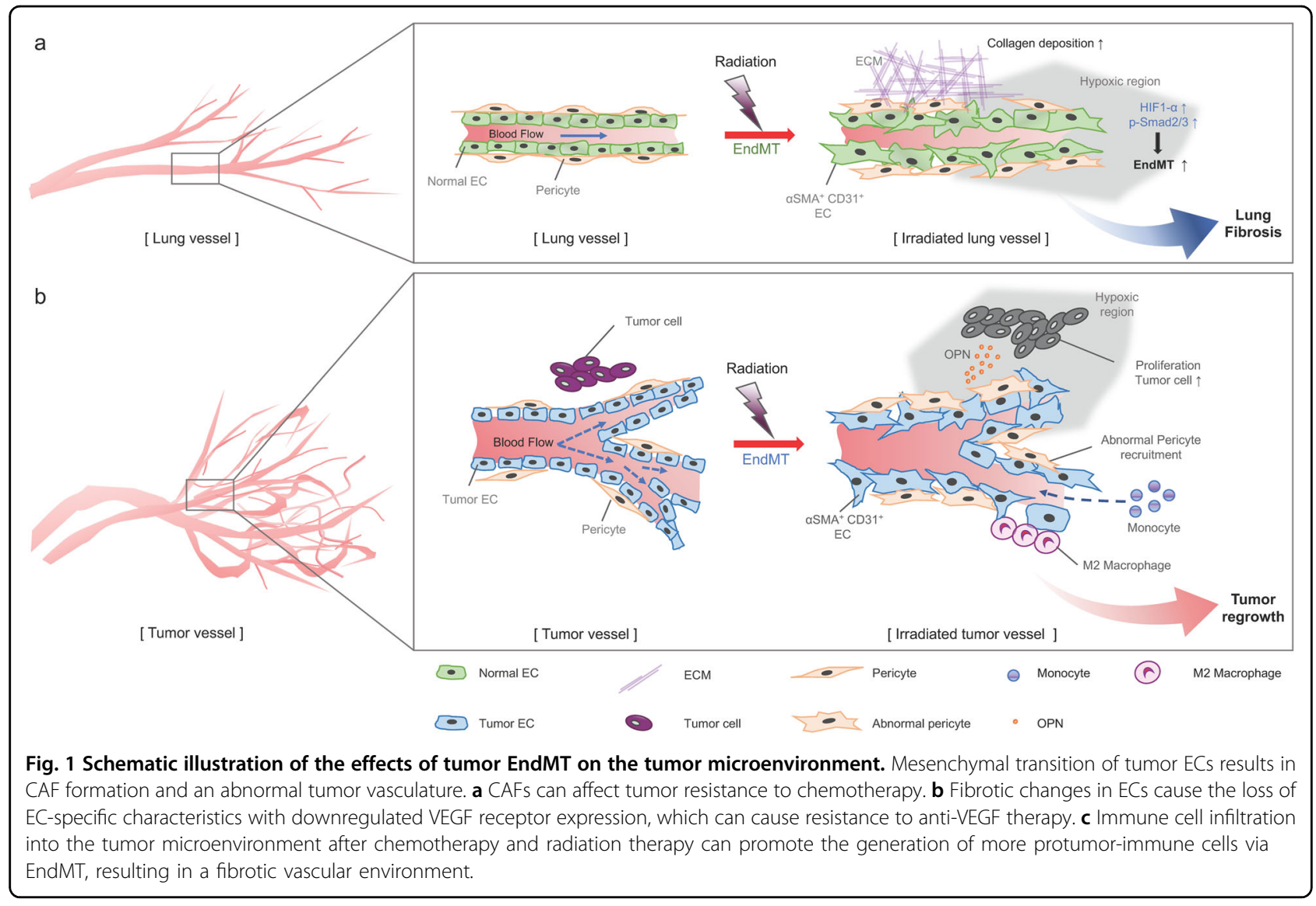

therapy are well known to cause cardiac toxicity. Recently, data from several studies demonstrated that EndMT occurs during the development of cardiac toxicity due to anticancer chemotherapies. A recent study showed that Dox and Herceptin increased drug permeability by affecting tight-junction formation by human cardiac microvascular-endothelial cells, showing decreased expression of ZO-1 and tight-junction protein-1, which resulted in cardiotoxicity ${ }^{23,78}$. Cardiotoxicity is also caused by monotherapy with Herceptin (trastuzumab), a humanized monoclonal antibody used to treat breast cancers.

Calcitriol, an active form of vitamin D3 that inhibits the growth of cancer cells, has been shown to attenuate Doxinduced myocardial fibrosis and fibrotic proteins, and improve diastolic function by reducing TGF- $\beta-S m a d 2-$ mediated EndMT and fibroblast-to-myofibroblast transition. The hearts of mice with EC-specific GFP expression showed increased levels of vimentin ${ }^{+} \mathrm{GFP}^{+}$ECs (indicative of EndMT), whereas calcitriol treatment attenuated these effects ${ }^{79}$. Administration of arsenic trioxide induced cardiac fibrosis in rats. In human aortic ECs, the AKT/ GSK-3 $\beta /$ cochlear pathway was activated in arsenic trioxide-mediated EndMT, and EndMT was inhibited by the AKT inhibitor LY294002 ${ }^{80}$.

\section{EndMT in radiation therapy}

Approximately half of all patients with cancer are treated with radiation therapy. Despite recent technological advances, tumor radioresistance and normal tissue damage remain challenges to improving cancer-cure rates. Normal tissue damage in radiation therapy remains a dose-limiting factor ${ }^{81}$.

In previous studies, we reported the occurrence of EndMT in irradiated tumor and normal tissues after radiation therapy ${ }^{18,61,82,83}$. Radiation-induced EndMT causes fibrotic changes in both tumors and the normal tissue microenvironment, such as in the lungs and heart vessels, which contribute to tumor radioresistance and normal tissue damage, respectively. Therefore, strategies targeting EndMT may enhance the efficacy of radiotherapy to overcome normal tissue damage and tumor radioresistance.

\section{Radiation-induced tumor EndMT}

There is increasing interest in combining radiotherapy with antiangiogenic therapy or immunotherapy to further improve the effectiveness of radiotherapy (Fig. 1) ${ }^{83}$.

Combining radiation therapy with antiangiogenic therapy has been reported to be effective, and VEGFR2 blockade may normalize the abnormal tumor vasculature 


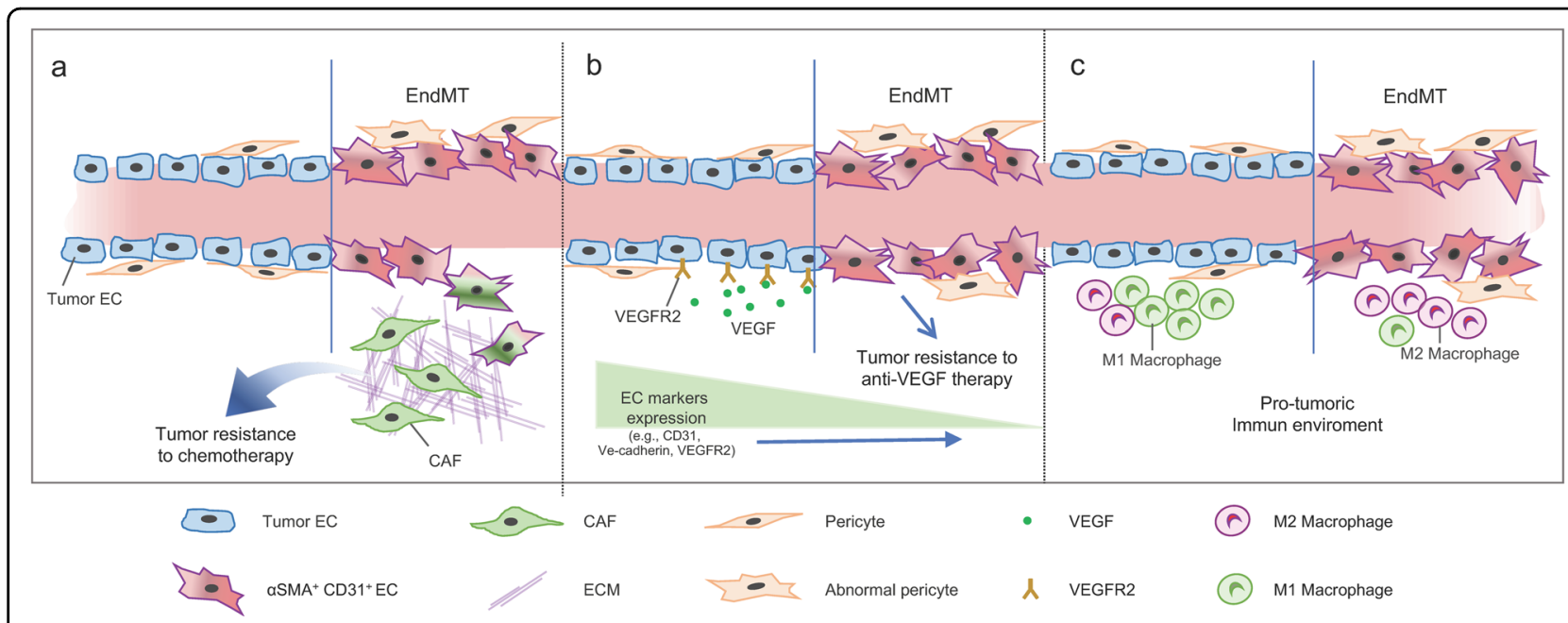

Fig. 2 Schematic illustration of radiation-induced EndMT during radiation therapy in patients with lung cancer. a Radiation-induced lung vascular damage causes the mesenchymal transition of lung ECs, and thus, hypoxic regions formed by damaged vessels enhance the process of tissue fibrosis. Increased HIF-a expression on vascular ECs enhances EndMT via Smad2/3 signaling. $\mathbf{b}$ In lung cancer, radioresistant tumors can regrow after radiation therapy. ECs remaining following irradiation undergo EndMT, which increases the tumor burden, and leads to the recruitment of abnormal pericytes. Tumor EndMT promotes tumor regrowth via EndMT-related secreted molecules (such as OPN), and tumor EndMT promotes M2 macrophage polarization of monocytes recruited into the tumor microenvironment after radiation therapy.

with increased pericyte and basement membrane coverage, resulting in tumor oxygenation and an increased response to radiation therapy ${ }^{84}$. Therefore, understanding the tumor endothelial transition in radiation therapy may lead to more efficient combined radiotherapies. Radiationinduced EndMT results in the formation of a tumor vasculature with irregular $\mathrm{SMA}^{+} \mathrm{NG}^{+}$pericyte recruitment in adenocarcinoma lung tumors with p53 deletion and KRAS mutation (Fig. 2). EndMT has also been observed in human non-small-cell lung cancer tissues following irradiation. EC-specific p53 deletion inhibits tumor EndMT and subsequent irregular $\mathrm{SMA}^{+} \mathrm{NG}^{+}$pericyte recruitment, resulting in decreased tumor regrowth after radiation therapy (Table 1). In addition, EC-specific TGFbR2 deletion in the tumor is associated with increased tumor regrowth after radiation therapy because of enhanced TGF $\beta R 1$ signaling (Table 1). Tumor EndMT occurring after radiotherapy results in osteopontin secretion, which stimulates $\mathrm{CD} 44 \mathrm{~V}^{+}$cancer stem cell proliferation, and contributes to the M2 polarization of macrophages. Coculture of monocytic cells and irradiated tumor ECs facilitated the conversion of monocytic cells into M2 macrophages ${ }^{18}$. In a neuroblastoma xenograft model, high-dose radiation therapy (HDRT) combined with Notch inhibition decreased tumor EC numbers more than HDRT alone. Notch inhibition reduced HDRT-induced EndMT, as demonstrated by decreased SMA-stained ECs. HDR increased Notch1 signaling, which was not observed at doses less than 2 Gy. These results suggest that Notch1 activation can protect tumor vessels and control the EndMT process in HDRT ${ }^{85}$.

\section{Radiation-induced vascular fibrosis}

During radiation therapy, radiation-induced tissue damage can cause morbidity, which is considered a treatment-limiting factor. In radiation-induced tissue damage, vascular fibrosis is well known as a prominent occurrence $^{86}$. Radiation directly induces DNA damage, and ionizing radiation causes ROS production and the activation of inflammatory processes, which result in fibrosis by increasing collagen deposition and reducing vascularity ${ }^{87}$. Blood vascular damage-induced tissue hypoxia and ischemia contribute to severe tissue injury, such as fibrosis and necrosis, and vascular fibrosis can greatly worsen the prognosis of patients undergoing radiotherapy ${ }^{81,88}$.

Irradiated ECs acquire a proinflammatory, procoagulant, and prothrombic phenotype, and increase the proliferation and migration of vascular smooth muscle cells ${ }^{88}$.

Radiation-induced vascular endothelium damage can lead to the burst release of ROS, and change the balance of angiogenesis, lipid-metabolism pathways, and immune homeostasis ${ }^{89}$. These acute effects can cause long-term vascular dysfunction ${ }^{89}$.

\section{Radiation-induced heart disease}

Cardiovascular complications limit the use of thoracic radiation therapy for treating Hodgkin lymphoma, lung cancer, and breast cancer ${ }^{90}$. A meta-analysis revealed excess mortality due to cardiac disease, including cardiovascular damage, in women undergoing radiation therapy for left-sided breast cancer ${ }^{91}$. Atherosclerosis occurs as a delayed side effect of radiation-induced heart disease ${ }^{92}$. 
Previously, we reported that radiation-induced EndMT occurs in human aortic ECs. Oxidized low-density lipoprotein increased radiation-induced EndMT, and irradiated $\mathrm{ApoE}^{-/-}$mice showed increased oxidized lowdensity lipoprotein levels and a more fibrotic phenotype of $\mathrm{ECs}^{82}$.

\section{Radiation-induced intestinal damage}

Gastrointestinal toxicity after radiotherapy is a major treatment-related complication. Radiation proctitis commonly occurs following inflammation of the rectal lining after treatment for cervical, prostate, and colon cancer. Recently, Mintet et al. showed that EndMT occurred during radiation proctitis development in Tie2GFP mice, and that tissue inflammation caused the phenotypic conversion of endothelial-to-mesenchymal cells $^{93}$. In conditional endothelial Hey2-deleted mice, EndMT occurrence and rectal tissue damage were reduced after irradiation. Microvascular protection reduced stem/clonogenic EC loss. These results suggest that EndMT can be targeted to mitigate radiationinduced intestinal damage (Table 1$)^{94}$.

\section{Radiation-induced pulmonary fibrosis (RIPF)}

Thoracic radiotherapy can cause RIPF as a late side effect. In particular, RIPF is one of the most frequent complications after radiotherapy for lung cancer. RIPF is characterized by fibroblast proliferation and leukocyte recruitment, as well as excessive extracellular matrix deposition. Previously, we reported that the presence of EndMT significantly increased during the early phase of RIPF development. In that study, it was demonstrated that initial vascular hypoxic damage induced EndMT at various irradiation doses in different tissue volumes. Vascular EndMT prominently appeared prior to EndMT of alveolar epithelial II cells ${ }^{38}$.

In irradiated human pulmonary artery ECs, EndMT was dependent on HIF- $1 \alpha$ expression via TGF- $\beta$ R1/Smad signaling. Data generated in that study revealed HIF-1 $\alpha$-related EndMT in human RIPF tissues ${ }^{95}$. A recent study provided evidence that in a coculture system of human fetal lung fibroblasts (MRC-5) and irradiated HUVECs, Snail and vimentin expression was upregulated, and CD31 expression was downregulated. Radiation-induced EndMT enhances the differentiation of fibroblasts to myofibroblasts via the Snail/ miR-199a-5p axis (Table 2) ${ }^{95}$.

\section{Drugs with EndMT-inhibiting effects}

Recently, several drugs in clinical testing have been reported to inhibit EndMT in various animal disease models (Table 1). These drugs inhibit EndMT by targeting various signaling molecules, such as DPP-4 ${ }^{25,96}, \mathrm{Smad}^{97}$, TGF- $\beta^{97-99}$, AMPK ${ }^{100}$, and other proteins ${ }^{28,101-106}$.

Nintedanib, which has been approved for treating pulmonary fibrosis, showed anti-vascular remodeling effects in pulmonary hypertension ${ }^{28}$. Macitentan was approved for treating pulmonary fibrosis and inhibited TGF- $\beta$ - and ET-1-mediated EndMT in ECs isolated from patients with systemic sclerosis ${ }^{98}$. Recent data demonstrated that diabetic complications were related to EndMT. High glucose levels can lead to EndMT, which is regulated by miRNAs (miR-200b and miR-18a-5p) (Table 2) ${ }^{107-110}$.

Vildagliptin, a drug used to treat diabetes, regulates DPP-4-dependent EndMT and showed anti-fibrotic effects in a mouse model of sepsis ${ }^{25}$. Liraglutide, linagliptin, and losartan, which have been approved for treating diabetes and its complications, inhibited EndMT in diabetic mice and diabetic complications in mouse models ${ }^{96,97,100}$. In addition, the GSK inhibitor, CHIR99021, was shown to inhibit radiation-induced EndMT in HUVECs ${ }^{101}$. Imatinib, a PDGF receptor antagonist, regulated EndMT in pulmonary artery remodeling of pulmonary artery hypertension in rats ${ }^{111}$. Table 3 shows various drugs that are used clinically to inhibit EndMT.

\section{Concluding remarks}

Taken together, the results of various studies have demonstrated that EndMT contributes to tissue fibrosis and cancer. EndMT can affect tumor progression via the mesenchymal transition of cells to CAFs, or effects on the abnormal tumor vasculature. Moreover, fibrotic changes and specific gene loss in tumor ECs may cause tumor resistance to and relapse after anti-VEGF therapy. Considering these tumor vasculature EndMT-related phenotypic changes, antiangiogenic therapies can be developed, particularly anti-VEGF therapy combined with chemo- and radiation therapy. The role of EndMT in regulating normal tissue damage, such as tissue fibrosis, should also be evaluated to reduce the side effects of anticancer therapy, such as cardiovascular damage, cardiotoxicity, and kidney and lung fibrosis. In particular, EndMT modulation may provide a new strategy for radiation therapy. Regulating EndMT in irradiated normal tissues around the tumor can reduce vascular damage and tissue fibrosis. Simultaneously, regulating tumor EndMT after radiation therapy may prevent tumor regrowth and tumor microenvironmental changes, such as those mediating the protumorigenic activities of immune cells in the fibrotic phase. Further studies are necessary to understand the exact mechanism behind tumor EndMT in human cancer.

Understanding EndMT may provide new therapeutic strategies against cancer, and reduce the related side effects, such as tissue damage.

\section{Acknowledgements}

This work was supported by grants from the National Research Foundation (NRF-2017M2A2A7A02019482, 2020R1A2B5B020027, and NRF-2017R1A2B2004156) and Korea Institute of Radiological \& Medical Sciences (KIRAMS, 50531-2020) funded by the Ministry of Science and ICT (MSIT), Republic of Korea. 


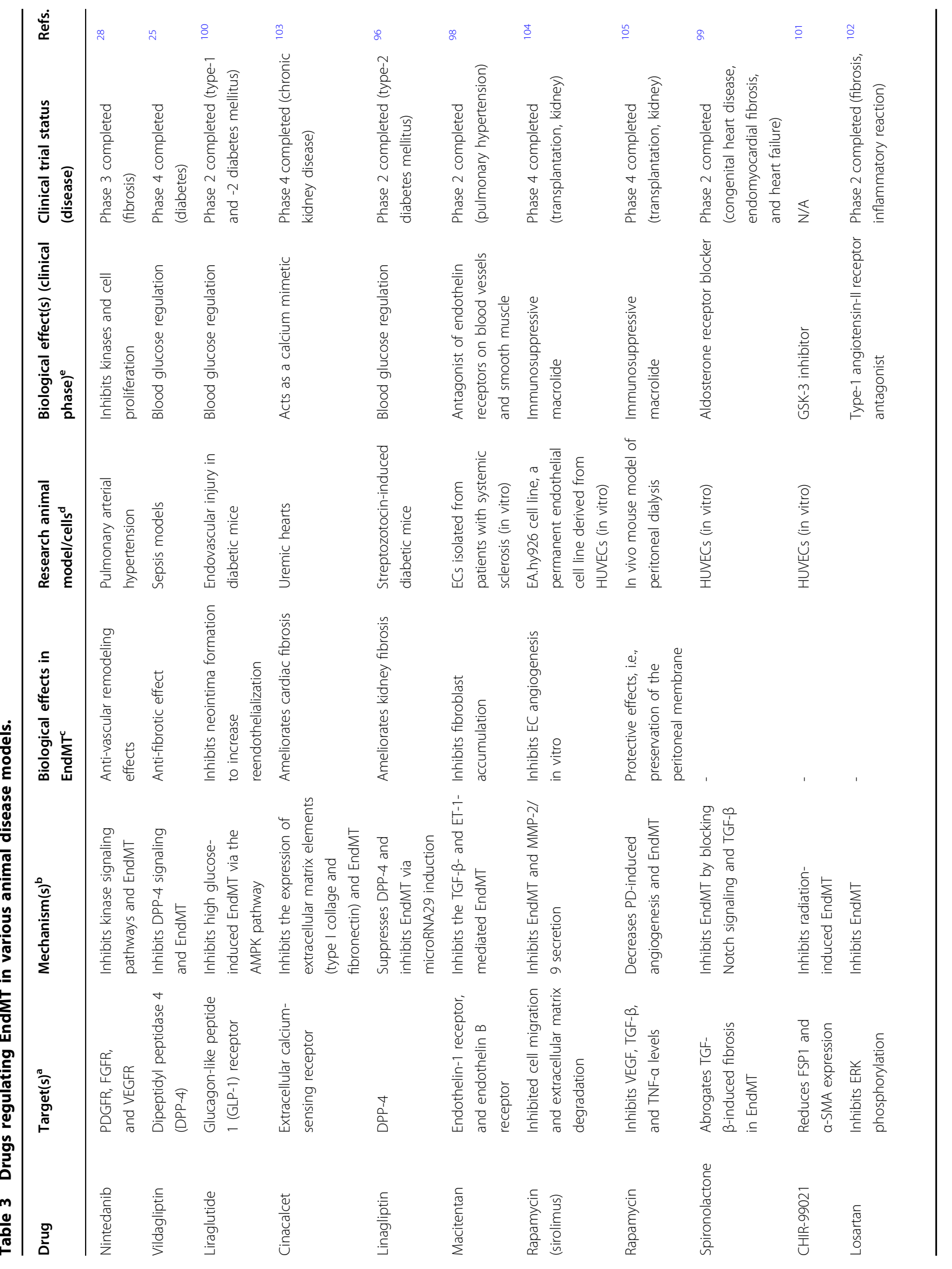




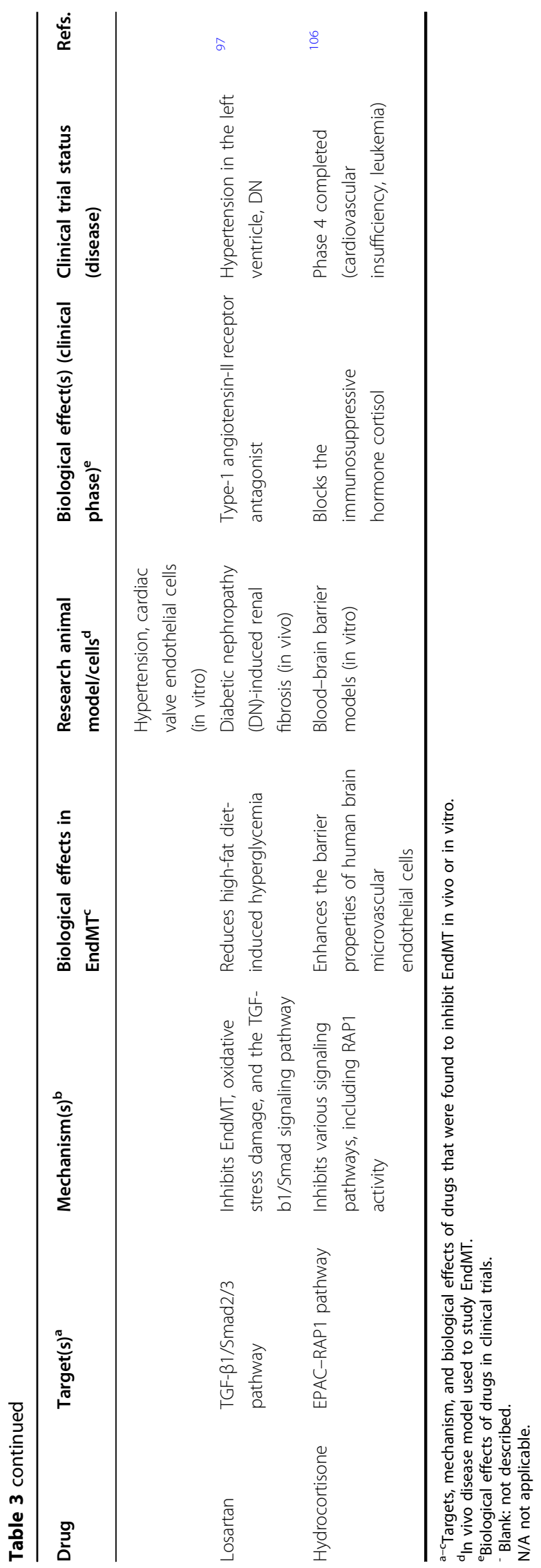

\section{Conflict of interest}

The authors declare that they have no conflict of interest.

\section{Publisher's note}

Springer Nature remains neutral with regard to jurisdictional claims in published maps and institutional affiliations.

Received: 14 February 2020 Revised: 28 March 2020 Accepted: 16 April 2020.

Published online: 28 May 2020

\section{References}

1. Cui, X. et al. Venous endothelial marker COUP-TFIl regulates the distinct pathologic potentials of adult arteries and veins. Sci. Rep. 5, 16193 (2015).

2. Krenning, G., Barauna, V. G., Krieger, J. E., Harmsen, M. C. \& Moonen, J. R. Endothelial plasticity: shifting phenotypes through force feedback. Stem Cells Int. 2016, 9762959 (2016).

3. Hong, L. et al. EndMT: a promising and controversial field. Eur. J. Cell Biol. 97, 493-500 (2018).

4. Platel, V., Faure, S., Corre, I. \& Clere, N. Endothelial-to-mesenchymal transition (EndoMT): roles in tumorigenesis, metastatic extravasation and therapy resistance. J. Oncol. 2019, 8361945 (2019).

5. Tinkelman, D. G. Theophylline-use and misuse in pediatric asthma. Hosp. Pract. 23, 179-184 (1988).

6. van Meeteren, L. A. \& ten Dijke, P. Regulation of endothelial cell plasticity by TGF-beta. Cell Tissue Res. 347, 177-186 (2012).

7. Zhong, A., Mirzaei, Z. \& Simmons, C. A. The roles of matrix stiffness and sscatenin signaling in endothelial-to-mesenchymal transition of aortic valve endothelial. Cells Cardiovasc. Eng. Technol. 9, 158-167 (2018).

8. Liu, J., Dong, F., Jeong, J., Masuda, T. \& Lobe, C. G. Constitutively active Notch1 signaling promotes endothelialmesenchymal transition in a conditional transgenic mouse model. Int J. Mol. Med. 34, 669-676 (2014).

9. Zhang, $H$. et al. Bone morphogenetic protein-7 inhibits endothelialmesenchymal transition in pulmonary artery endothelial cell under hypoxia. J. Cell Physiol. 233, 4077-4090 (2018).

10. Guo, Y. et al. Kallistatin inhibits TGF-beta-induced endothelial-mesenchymal transition by differential regulation of microRNA-21 and eNOS expression. Exp. Cell Res. 337, 103-110 (2015).

11. Song, S. et al. Foxm 1 is a critical driver of TGF-beta-induced EndMT in endothelial cells through Smad2/3 and binds to the Snail promoter. J. Cell Physiol. 234, 9052-9064 (2019).

12. Mahmoud, M. M. et al. Shear stress induces endothelial-to-mesenchymal transition via the transcription factor Snail. Sci. Rep. 7, 3375 (2017).

13. Saito, A. EMT and EndMT: regulated in similar ways? J. Biochem. 153 493-495 (2013).

14. Fiori, M. E. et al. Cancer-associated fibroblasts as abettors of tumor progression at the crossroads of EMT and therapy resistance. Mol. Cancer 18, 70 (2019).

15. Kalluri, R. \& Zeisberg, M. Fibroblasts in cancer. Nat. Rev. Cancer 6, 392-401 (2006)

16. Huang, L., Nakayama, H., Klagsbrun, M., Mulliken, J. B. \& Bischoff, J. Glucose transporter 1-positive endothelial cells in infantile hemangioma exhibit features of facultative stem cells. Stem Cells 33, 133-145 (2015).

17. Moonen, J. R. et al. Endothelial progenitor cells give rise to pro-angiogenic smooth muscle-like progeny. Cardiovasc Res. 86, 506-515 (2010).

18. Choi, S. H. et al. Tumour-vasculature development via endothelial-tomesenchymal transition after radiotherapy controls CD44v6(+) cancer cell and macrophage polarization. Nat. Commun. 9, 5108 (2018).

19. Huang, M. et al. C-Met-mediated endothelial plasticity drives aberrant vascularization and chemoresistance in glioblastoma. J. Clin. Invest. 126, 1801-1814 (2016).

20. Zeisberg, E. M. et al. Endothelial-to-mesenchymal transition contributes to cardiac fibrosis. Nat. Med. 13, 952-961 (2007).

21. Cooley, B. C. et al. TGF-beta signaling mediates endothelial-to-mesenchymal transition (EndMT) during vein graft remodeling. Sci. Transl. Med. 6, 227ra234 (2014).

22. Widyantoro, B. et al. Endothelial cell-derived endothelin-1 promotes cardiac fibrosis in diabetic hearts through stimulation of endothelial-tomesenchymal transition. Circulation 121, 2407-2418 (2010). 
23. $\mathrm{Xu}, \mathrm{X}$. et al. Snail is a direct target of hypoxia-inducible factor 1alpha (HIF1alpha) in hypoxia-induced endothelial to mesenchymal transition of human coronary endothelial cells. J. Biol. Chem. 290, 16653-16664 (2015).

24. Zhang, B. et al. Hypoxia induces endothelialmesenchymal transition in pulmonary vascular remodeling. Int. J. Mol. Med. 42, 270-278 (2018).

25. Suzuki, T. et al. Vildagliptin ameliorates pulmonary fibrosis in lipopolysaccharide-induced lung injury by inhibiting endothelial-tomesenchymal transition. Respir. Res. 18, 177 (2017).

26. Nataraj, D., Ernst, A. \& Kalluri, R. Idiopathic pulmonary fibrosis is associated with endothelial to mesenchymal transition. Am. J. Respir. Cell Mol. Biol. 43, 129-130 (2010).

27. Hashimoto, N. et al. Endothelial-mesenchymal transition in bleomycininduced pulmonary fibrosis. Am. J. Respir. Cell Mol. Biol. 43, 161-172 (2010).

28. Tsutsumi, T. et al. Nintedanib ameliorates experimental pulmonary arterial hypertension via inhibition of endothelial mesenchymal transition and smooth muscle cell proliferation. PLOS ONE 14, e0214697 (2019).

29. Yan, F. et al. Glucagon-Like peptide 1 protects against hyperglycemicinduced endothelial-to-mesenchymal transition and improves myocardial dysfunction by suppressing poly(ADP-Ribose) polymerase 1 activity. Mol. Med. 21, 15-25 (2015).

30. Montorfano, I. et al. Oxidative stress mediates the conversion of endothelial cells into myofibroblasts via a TGF-beta1 and TGF-beta2-dependent pathway. Lab. Invest. 94, 1068-1082 (2014).

31. Pociask, D. A., Sime, P. J. \& Brody, A. R. Asbestos-derived reactive oxygen species activate TGF-beta1. Lab. Invest. 84, 1013-1023 (2004).

32. Ahmed, S. M., Luo, L., Namani, A., Wang, X. J. \& Tang, X. Nrf2 signaling pathway: pivotal roles in inflammation. Biochim. Biophys. Acta Mol. Basis Dis. 1863, 585-597 (2017).

33. Good, R. B. et al. Endothelial to mesenchymal transition contributes to endothelial dysfunction in pulmonary arterial hypertension. Am. J. Pathol. 185, 1850-1858 (2015).

34. Rieder, F. et al. Inflammation-induced endothelial-to-mesenchymal transition: a novel mechanism of intestinal fibrosis. Am. J. Pathol. 179, 2660-2673 (2011).

35. Potenta, S., Zeisberg, E. \& Kalluri, R. The role of endothelial-to-mesenchymal transition in cancer progression. Br. J. Cancer 99, 1375-1379 (2008).

36. Brush, J. et al. Molecular mechanisms of late normal tissue injury. Semin. Radiat. Oncol. 17, 121-130 (2007).

37. Rodemann, H. P. \& Bamberg, M. Cellular basis of radiation-induced fibrosis. Radiother. Oncol. 35, 83-90 (1995).

38. Choi, S. H. et al. A hypoxia-induced vascular endothelial-to-mesenchyma transition in development of radiation-induced pulmonary fibrosis. Clin. Cancer Res. 21, 3716-3726 (2015).

39. Evrard, S. M. et al. Endothelial to mesenchymal transition is common in atherosclerotic lesions and is associated with plaque instability. Nat. Commun. 7, 11853 (2016)

40. Chen, Y. et al. Activation of Nrf2 attenuates pulmonary vascular remodeling via inhibiting endothelial-to-mesenchymal transition: an insight from a plant polyphenol. Int. J. Biol. Sci. 13, 1067-1081 (2017).

41. Anea, C. B. et al. Increased superoxide and endothelial NO synthase uncoupling in blood vessels of Bmal1-knockout mice. Circ. Res. 111 1157-1165 (2012).

42. Khapre, R. V., Kondratova, A. A., Susova, O. \& Kondratov, R. V. Circadian clock protein BMAL1 regulates cellular senescence in vivo. Cell Cycle 10, 4162-4169 (2011).

43. Zhu, M. et al. BMAL1 suppresses ROS-induced endothelial-to-mesenchymal transition and atherosclerosis plaque progression via BMP signaling. Am. J. Transl. Res. 10, 3150-3161 (2018).

44. Li, J. et al. Low-intensity pulsed ultrasound prevents the oxidative stress induced endothelial-mesenchymal transition in human aortic endothelial cells. Cell Physiol. Biochem. 45, 1350-1365 (2018).

45. Lee, E. S., Boldo, L. S., Fernandez, B. O., Feelisch, M. \& Harmsen, M. C. Suppression of TAK1 pathway by shear stress counteracts the inflammatory endothelial cell phenotype induced by oxidative stress and TGF-beta1. Sci. Rep. 7, 42487 (2017).

46. Maleszewska, M. et al. IL-1 beta and TGFbeta2 synergistically induce endothelial to mesenchymal transition in an NFkappaB-dependent manner Immunobiology 218, 443-454 (2013).

47. Jiang, F., Liu, G. S., Dusting, G. J. \& Chan, E. C. NADPH oxidase-dependent redox signaling in TGF-beta-mediated fibrotic responses. Redox Biol. 2 267-272 (2014).
48. You, S. et al. Schizandrin B attenuates angiotensin II induced endothelial to mesenchymal transition in vascular endothelium by suppressing NF-kappaB activation. Phytomedicine 62, 152955 (2019).

49. Xu, Y. P. et al. MiR-126a-5p is involved in the hypoxia-induced endothelial-tomesenchymal transition of neonatal pulmonary hypertension. Hypertens. Res. 40, 552-561 (2017).

50. Watson, C. J. et al. Hypoxia-induced epigenetic modifications are associated with cardiac tissue fibrosis and the development of a myofibroblast-like phenotype. Hum. Mol. Genet. 23, 2176-2188 (2014).

51. Zou, J. et al. Autophagy attenuates endothelial-to-mesenchymal transition by promoting Snail degradation in human cardiac microvascular endothelial cells. Biosci. Rep. 37, BSR20171049 (2017)

52. Zeisberg, E. M., Potenta, S., Xie, L., Zeisberg, M. \& Kalluri, R. Discovery of endothelial to mesenchymal transition as a source for carcinoma-associated fibroblasts. Cancer Res. 67, 10123-10128 (2007).

53. Zhu, K. et al. MiR-302c inhibits tumor growth of hepatocellular carcinoma by suppressing the endothelial-mesenchymal transition of endothelial cells. Sci. Rep. 4, 5524 (2014).

54. Nie, L. et al. Endothelial-mesenchymal transition in normal human esophageal endothelial cells cocultured with esophageal adenocarcinoma cells: role of IL-1 beta and TGF-beta2. Am. J. Physiol. Cell Physiol. 307, C859-C877 (2014).

55. Garcia, J. et al. Tie1 deficiency induces endothelial-mesenchymal transition. EMBO Rep. 13, 431-439 (2012).

56. Noseda, M. et al. Notch activation results in phenotypic and functional changes consistent with endothelial-to-mesenchymal transformation. Circ. Res. 94, 910-917 (2004).

57. Timmerman, L. A. et al. Notch promotes epithelial-mesenchymal transition during cardiac development and oncogenic transformation. Genes Dev. 18, 99-115 (2004).

58. Blokzijl, A. et al. Cross-talk between the Notch and TGF-beta signaling pathways mediated by interaction of the Notch intracellular domain with Smad3. J. Cell Biol. 163, 723-728 (2003).

59. Zavadil, J., Cermak, L., Soto-Nieves, N. \& Bottinger, E. P. Integration of TGFbeta/Smad and Jagged1/Notch signalling in epithelial-to-mesenchyma transition. EMBO J. 23, 1155-1165 (2004).

60. Ghiabi, P. et al. Breast cancer cells promote a notch-dependent mesenchymal phenotype in endothelial cells participating to a pro-tumoral niche. J. Transl. Med. 13, 27 (2015)

61. Choi, S. H. et al. HSPB1 inhibits the endothelial-to-mesenchymal transition to suppress pulmonary fibrosis and lung tumorigenesis. Cancer Res. 76, 1019-1030 (2016).

62. Fan, C. S. et al. Osteopontin-integrin engagement induces HIF-1alpha-TCF12mediated endothelial-mesenchymal transition to exacerbate colorectal cancer. Oncotarget 9, 4998-5015 (2018).

63. Kim, S. H., Song, Y. \& Seo, H. R. GSK-3beta regulates the endothelial-tomesenchymal transition via reciprocal crosstalk between NSCLC cells and HUVECs in multicellular tumor spheroid models. J. Exp. Clin. Cancer Res. 38, 46 (2019).

64. Wu, D. M. et al. PLEK2 mediates metastasis and vascular invasion via the ubiquitin-dependent degradation of SHIP2 in non-small cell lung cancer. Int J. Cancer 146, 2563-2575 (2019).

65. Dudley, A. C. Tumor endothelial cells. Cold Spring Harb. Perspect. Med. 2, a006536 (2012)

66. Sun, H., Breslin, J. W., Zhu, J., Yuan, S. Y. \& Wu, M. H. Rho and ROCK signaling in VEGF-induced microvascular endothelial hyperpermeability. Microcirculation 13, 237-247 (2006).

67. Fujii, M. et al. Inhibition of Rho kinase by hydroxyfasudil attenuates brain edema after subarachnoid hemorrhage in rats. Neurochem. Int. 60, 327-333 (2012).

68. Li, B. et al. Involvement of Rho/ROCK signalling in small cell lung cancer migration through human brain microvascular endothelial cells. FEBS Lett. 580, 4252-4260 (2006)

69. Nagai, N. et al. Downregulation of ERG and FLI1 expression in endothelial cells triggers endothelial-to-mesenchymal transition. PLOS Genet. 14, e1007826 (2018).

70. Chan, T. S. et al. Metronomic chemotherapy prevents therapy-induced stromal activation and induction of tumor-initiating cells. J. Exp. Med. 213, 2967-2988 (2016).

71. Su, S. et al. CD10(+)GPR77(+) cancer-associated fibroblasts promote cancer formation and chemoresistance by sustaining cancer stemness. Cell $\mathbf{1 7 2}$ 841-856 e816 (2018). 
72. Prakash, J. Cancer-associated fibroblasts: perspectives in cancer therapy. Trends Cancer 2, 277-279 (2016).

73. Liu, T. et al. PDGF-mediated mesenchymal transformation renders endothelial resistance to anti-VEGF treatment in glioblastoma. Nat. Commun. 9 3439 (2018).

74. Helfrich, l. et al. Resistance to antiangiogenic therapy is directed by vascular phenotype, vessel stabilization, and maturation in malignant melanoma. J. Exp. Med. 207, 491-503 (2010).

75. Franco, M., Paez-Ribes, M., Cortez, E., Casanovas, O. \& Pietras, K. Use of a mouse model of pancreatic neuroendocrine tumors to find pericyte biomarkers of resistance to anti-angiogenic therapy. Horm. Metab. Res. 43 , 884-889 (2011).

76. Xian, X. et al. Pericytes limit tumor cell metastasis. J. Clin. Invest. 116, 642-651 (2006).

77. Anderberg, C. et al. Deficiency for endoglin in tumor vasculature weakens the endothelial barrier to metastatic dissemination. J. Exp. Med. 210, 563-579 (2013).

78. Wilkinson, E. L., Sidaway, J. E. \& Cross, M. J. Cardiotoxic drugs Herceptin and doxorubicin inhibit cardiac microvascular endothelial cell barrier formation resulting in increased drug permeability. Biol. Open 5, 1362-1370 (2016).

79. Tsai, T. H., Lin, C. J., Hang, C. L. \& Chen, W. Y. Calcitriol attenuates doxorubicininduced cardiac dysfunction and inhibits endothelial-to-mesenchymal transition in mice. Cells 8, 865 (2019).

80. Zhang, Y. et al. Endothelial to mesenchymal transition contributes to arsenictrioxide-induced cardiac fibrosis. Sci. Rep. 6, 33787 (2016).

81. Stone, H. B., Coleman, C. N., Anscher, M. S. \& McBride, W. H. Effects of radiation on normal tissue: consequences and mechanisms. Lancet Oncol. 4, 529-536 (2003).

82. Kim, M. et al. The effect of oxidized low-density lipoprotein (ox-LDL) on radiation-induced endothelial-to-mesenchymal transition. Int J. Radiat. Biol. 89, 356-363 (2013)

83. Goedegebuure, R. S. A., de Klerk, L. K., Bass, A. J., Derks, S. \& Thijssen, V. Combining radiotherapy with anti-angiogenic therapy and immunotherapy; a therapeutic triad for cancer? Front. Immunol. 9, 3107 (2018).

84. Winkler, F. et al. Kinetics of vascular normalization by VEGFR2 blockade governs brain tumor response to radiation: role of oxygenation, angiopoietin-1, and matrix metalloproteinases. Cancer Cell 6, 553-563 (2004).

85. Banerjee, D. et al. High-dose radiation increases Notch1 in tumor vasculature. Int J. Radiat. Oncol. Biol. Phys. 106, 857-866 (2019).

86. Weintraub, N. L., Jones, W. K. \& Manka, D. Understanding radiation-induced vascular disease. J. Am. Coll. Cardiol. 55, 1237-1239 (2010)

87. Straub, J. M. et al. Radiation-induced fibrosis: mechanisms and implications for therapy. J. Cancer Res. Clin. Oncol. 141, 1985-1994 (2015).

88. Dorresteijn, L. D. et al. Increased risk of ischemic stroke after radiotherapy on the neck in patients younger than 60 years. J. Clin. Oncol. 20, 282-288 (2002)

89. Venkatesulu, B. P. et al. Radiation-induced endothelial vascular injury: a review of possible mechanisms. JACC Basic Transl. Sci. 3, 563-572 (2018).

90. Moslehi, J. J. Cardiovascular toxic effects of targeted cancer therapies. N. Engl. J. Med. 375, 1457-1467 (2016).

91. Darby, S. C. et al. Risk of ischemic heart disease in women after radiotherapy for breast cancer. N. Engl. J. Med. 368, 987-998 (2013).

92. Boerma, M. \& Hauer-Jensen, M. Potential targets for intervention in radiationinduced heart disease. Curr. Drug Targets 11, 1405-1412 (2010).

93. Mintet, E. et al. Identification of endothelial-to-mesenchymal transition as a potential participant in radiation proctitis. Am. J. Pathol. 185, 2550-2562 (2015)
94. Mintet, E. et al. Endothelial Hey2 deletion reduces endothelial-tomesenchymal transition and mitigates radiation proctitis in mice. Sci. Rep. 7, 4933 (2017)

95. Yi, M. et al. Irradiated human umbilical vein endothelial cells undergo endothelial-mesenchymal transition via the Snail/miR-199a-5p axis to promote the differentiation of fibroblasts into myofibroblasts. Biomed. Res. Int. 2018, 4135806 (2018).

96. Kanasaki, K. et al. Linagliptin-mediated DPP-4 inhibition ameliorates kidney fibrosis in streptozotocin-induced diabetic mice by inhibiting endothelial-tomesenchymal transition in a therapeutic regimen. Diabetes 63, 2120-2131 (2014)

97. Yao, Y. et al. Losartan alleviates renal fibrosis and inhibits endothelial-tomesenchymal transition (EMT) under high-fat diet-induced hyperglycemia. Front. Pharm. 9, 1213 (2018).

98. Cipriani, P et al. The endothelial-mesenchymal transition in systemic sclerosis is induced by endothelin-1 and transforming growth factor-beta and may be blocked by macitentan, a dual endothelin-1 receptor antagonist. J. Rheumatol. 42, 1808-1816 (2015).

99. Chen, X. et al. Protective effect of spironolactone on endothelial-tomesenchymal transition in HUVECs via Notch pathway. Cell Physiol. Biochem. 36, 191-200 (2015)

100. Tsai, T. H. et al. Liraglutide inhibits endothelial-to-mesenchymal transition and attenuates neointima formation after endovascular injury in streptozotocininduced diabetic mice. Cells 8, 589 (2019).

101. Song, Y. et al. Identification of radiation-induced EndMT inhibitors through cell-based phenomic screening. FEBS Open Bio 9, 82-91 (2019).

102. Wylie-Sears, J., Levine, R. A. \& Bischoff, J. Losartan inhibits endothelial-tomesenchymal transformation in mitral valve endothelial cells by blocking transforming growth factor-beta-induced phosphorylation of ERK. Biochem. Biophys. Res. Commun. 446, 870-875 (2014).

103. Wu, M. et al. Cinacalcet ameliorates cardiac fibrosis in uremic hearts through suppression of endothelial-to-mesenchymal transition. Int. J. Cardiol. 171 e65-e69 (2014).

104. Gao, H., Zhang, J., Liu, T. \& Shi, W. Rapamycin prevents endothelial cel migration by inhibiting the endothelial-to-mesenchymal transition and matrix metalloproteinase-2 and -9: an in vitro study. Mol. Vis. 17, 3406-3414 (2011).

105. Gonzalez-Mateo, G. T. et al. Rapamycin protects from type-i peritoneal membrane failure inhibiting the angiogenesis, lymphangiogenesis, and endo-MT. Biomed. Res. Int. 2015, 989560 (2015).

106. Furihata, T. et al. Hydrocortisone enhances the barrier properties of HBMEC/ cibeta, a brain microvascular endothelial cell line, through mesenchymal-toendothelial transition-like effects. Fluids Barriers CNS 12, 7 (2015).

107. Cao, Y, Feng, B, Chen, S, Chu, Y. \& Chakrabarti, S. Mechanisms of endothelia to mesenchymal transition in the retina in diabetes. Invest. Ophthalmol. Vis. Sci. 55, 7321-7331 (2014)

108. Feng, B. et al. miR-200b mediates endothelial-to-mesenchymal transition in diabetic cardiomyopathy. Diabetes 65, 768-779 (2016).

109. Kumarswamy, R. et al. Transforming growth factor-beta-induced endothelialto-mesenchymal transition is partly mediated by microRNA-21. Arterioscler. Thromb. Vasc. Biol, 32, 361-369 (2012).

110. Geng, H. \& Guan, J. MiR-18a-5p inhibits endothelial-mesenchymal transition and cardiac fibrosis through the Notch2 pathway. Biochem. Biophys. Res. Commun. 491, 329-336 (2017).

111. Song, S. et al. The role of PDGF-B/TGF-beta1/neprilysin network in regulating endothelial-to-mesenchymal transition in pulmonary artery remodeling. Cell Signal. 28, 1489-1501 (2016). 